\title{
Occult uterine leiomyosarcoma in women undergoing abdominal and minimally invasive surgeries for myomas
}

\author{
Artur Ludwin, Iwona Gawron, Kazimierz Pityński \\ Department of Gynecology and Oncology, Jagiellonian University, Krakow, Poland
}

\begin{abstract}
Objectives: To estimate (i) the incidence of occult uterine leiomyosarcoma (LMS) in patients operated on for presumed myomas, and (ii) the proportion of occult LMS to preoperatively diagnosed LMS in a tertiary center.

Material and methods: An Institutional Review Board-approved retrospective cohort study was performed. The electronic database of 30,476 patients was searched for women who had undergone surgery due to presumed myomas $(\mathrm{N}=2675)$ as well as those with uterine LMS recognized via histology $(N=10)$ between January 2010 and December 2016.

Results: Six of the 2675 treated women had occult LMS (incidence 1:446; 0.002; $\mathrm{Cl} 0.0-0.013$ ), and one underwent power morcellation (incidence $1: 951 ; 0.001 ; \mathrm{Cl} 0.0-0.006)$. Parallel searching revealed that 6 of the 10 cases $(60 \%)$ with uterine LMS recognized via histology were diagnosed postoperatively, whereas 4 of the $10(40 \%)$ were diagnosed preoperatively. The incidence of LMS morcellation during laparoscopy was 1:951 and, when all MIS cases were included, 1:1178. The patient who underwent LMS morcellation was operated in the general surgery ward 5 years after laparoscopy (omental recurrence).

Conclusions: These results are similar to the first and recent conservative FDA estimations, but two-times lower for procedures with laparoscopic morcellation and all MIS procedures than for abdominal. Because above half of LMS may be recognized after surgery, the risk of occult LMS and the delay of targeted surgical treatment should be included in all informed consent forms for conservative management of presumed myomas without histology.
\end{abstract}

Key words: leiomyosarcoma, laparoscopic morcellation, myomectomy, hysterectomy

Ginekologia Polska 2018; 89, 10: 546-552

\section{INTRODUCTION}

After a well-educated injured patient pointed out the underestimation of occult malignancy risk and questioned the safety of power morcellation, a polarizing debate [1-3] began which resulted in the first estimation of the prevalence of occult LMS by the Food and Drug Administration (FDA) [4] and a significant restriction in the use of morcellators in clinical practice in the United States. The prevalence of unexpected LMS varies from 1:495 to 1:1100, by FDA estimations, to $1: 8000$, depending on the analytical method used $[5,6]$. Many reports selectively focus on specific laparoscopic procedures, without showing the prevalence of occult malignancy in other myoma surgery procedures, nor determining the proportion of occult LMS to all cases of recognized LMS [7].
The main purposes of this study were to estimate (i) the incidence of occult uterine LMS in patients operated on for presumed fibroids, and (ii) the proportion of occult LMS diagnosed preoperatively. The secondary aims were to determine the incidence of uterine LMS during minimally invasive surgery (MIS) techniques, including high-power morcellation. Moreover, the incidence of occult LMS was analyzed in relation to specific procedures with morcellation and in relation to preselection for the following two settings: the MIS unit versus the gynecologic oncology unit and abdominal surgery versus MIS. Finally, the incidence of occult LMS was compared between MIS and traditional approach cases in a tertiary teaching hospital. 


\section{MATERIAL AND METHODS}

Design and patients

This was a retrospective observational study performed on datasets gathered in a tertiary referral and teaching center, in the Department of Gynecology and Oncology and the Department of Pathomorphology at Jagiellonian University, Cracow, Poland. Data collection began after the approval of the Jagiellonian University-Medical College Institutional Review Board (No 122.6120.42.2017). Firstly, the Department of Pathomorphology digital archives were searched for the pathological diagnosis of "leiomyosarcoma". The specimen provider was limited to the Department of Gynecology and Oncology, and the search was limited to the period between January 1, 2010 and December 31, 2016. The inclusion criterion was uterine LMS. At the same time, all surgical procedures performed for presumed fibroids were registered to establish the prevalence of LMS in the study population and the risk of LMS morcellation during surgeries performed for presumed fibroids. Records were classified with respect to the surgical approach-abdominal surgery versus MIS (laparoscopy and other vaginal procedures, including hysteroscopy). Moreover, procedures were divided into myomectomy and hysterectomy, and laparoscopic procedures with the use of power morcellation were counted. All consecutive patients undergoing surgery for presumed myomas were included in the study. Medical, pathological and surgical reports were reviewed to confirm indications, procedures and pathological diagnoses.

Subsequently, medical records (traditional and digital) of women diagnosed with LMS were systematically reviewed in terms of clinical characteristics and surgical approach. The data on patient demographics (age, body mass index, gravidity and parity, hormonal status, medical past history of malignancies and surgeries), reported symptoms, ultrasound findings (number of uterine masses and diameter of the largest mass), possible endometrial histopathological verification, surgical management (type of primary surgery, use of minimally invasive techniques, application of high-power morcellation) and, finally, tumor characteristics (grade and stage) were retrieved from clinical dossiers, ultrasound printouts and pathological reports. This parallel record search methodology ('LMS', surgery of 'presumed myomas') was designed to estimate the incidence of occult LMS in presumed myoma surgeries and the proportion of occult LMS to preoperatively recognized LMS cases.

\section{Settings}

During the study period, the Department of Gynecology and Oncology consisted of two surgical units, Gynecologic Oncology and Endoscopic Gynecology (or MIS unit), in addition to Chemotherapy and Brachytherapy units. The two surgical units with two operating theatres performed the sur- gical treatment of selected female populations. The gynecologic oncology unit was targeted for the treatment of patients with cancer, especially when extended abdominal surgery was planned or contraindications to MIS were present.

\section{Patient management}

An attending obstetrician-gynecologist or gynecologist-oncologist (more than 15 doctors in the study period) conducted qualifications for surgery. The gynecological examination, including vaginal speculum and bimanual examination, transvaginal sonography and colposcopy, and 3D sonohysterography (when needed) [8], and detailed counseling were performed. All women had a Pap smear test done no earlier than one year prior to surgery. All operations were performed by attendings or residents under the supervision of a participating attending physician.

\section{Statistical analysis}

The incidence of occult LMS during myoma surgery and data for other important endpoints (power morcellation, laparoscopy, laparoscopic and abdominal myomectomy) is shown with $95 \%$ confidence intervals (Cls) using the Wilson method recommended for use with a small $n$ [9]. The relative risk (RR) of occult LMS in MIS cases and the $95 \% \mathrm{CI}$ [10] were calculated [11]. Using the test of significance, the $P$-value was calculated [12]. Statistical significance was defined as $P \leq 0.05$. All calculations were performed using Statistica version 10.0 software (StatSoft Inc., Tulsa, OK, USA).

\section{RESULTS \\ Main findings}

Within the seven-year study period (January 2010 to December 2016), a total of 30,476 patients were hospitalized in the included departments, of which 15,348 were subjected to surgical treatment in the operating theatres -7232 in the gynecologic oncology unit and 8116 in MIS unit; 2675 cases (mean age \pm SD: 47 years \pm 9.4 , median 46, lower-upper quartile: 42-52) underwent surgery for presumed fibroids - 1712 in the MIS unit and 963 in the gynecologic oncology unit. The flow diagram in Figure 1 shows the specific surgeries and occult LMS incidence. There were 6 cases of occult LMS discovered (incidence 1:446; 0.002; $\mathrm{Cl} 0.0-0.013$ ); one underwent power morcellation (incidence $1: 951 ; 0.001 ; \mathrm{Cl} 0.0-0.006$ ).

\section{Proportion of occult LMS to preoperatively diagnosed LMS cases}

Data from the Department of Pathomorphology revealed 12 cases of LMS - ten uterine and two ovarian. Four of the ten (40\%) uterine LMS were diagnosed preoperatively, whereas six out of ten (60\%) were diagnosed postoperatively (Fig. 2-4). 


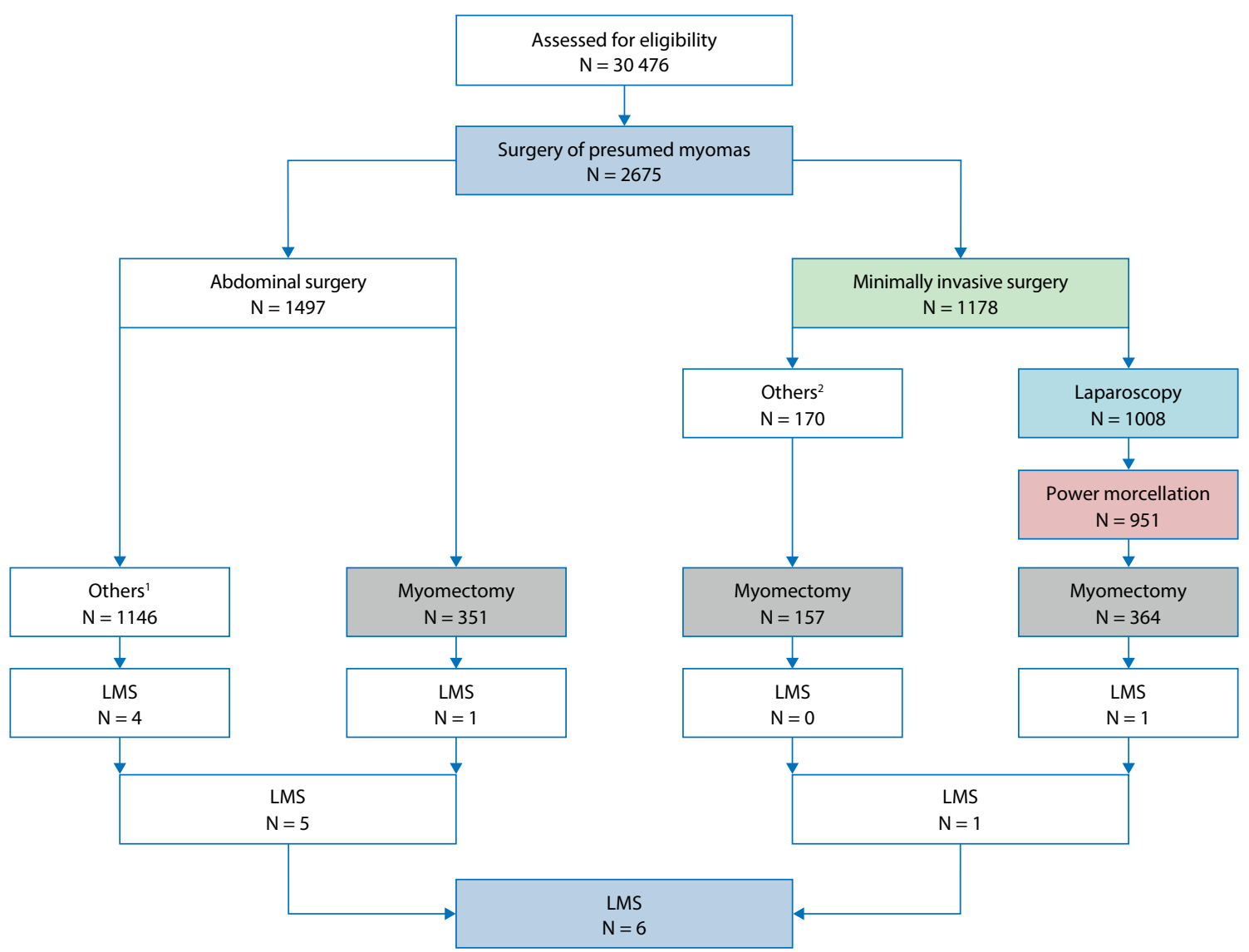

Figure 1. Study flow diagram

${ }^{1}$ total and supracervical hysterectomy;

${ }^{2}$ hysteroscopic myomectomy, vaginal myomectomy and hysterectomy

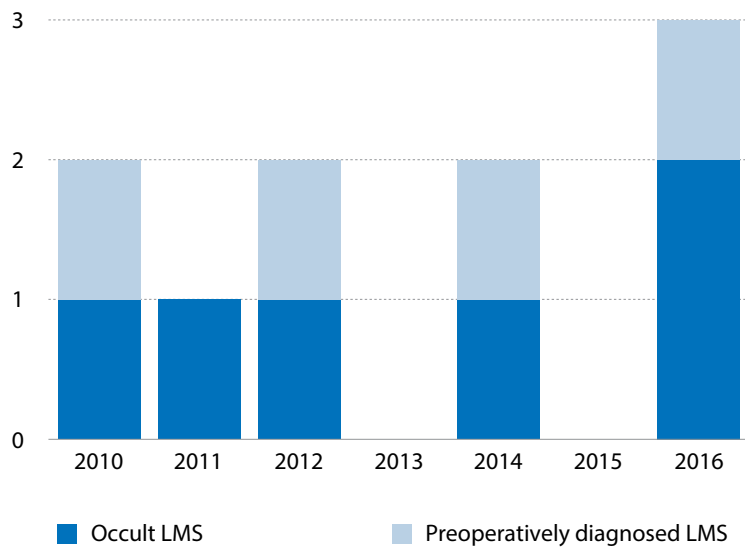

Figure 2. Proportion of occult to preoperatively diagnosed LMS by the year of recognition

\section{Preselection for MIS and Gynecologic Oncology units}

Comparative assessment of the two units (MIS vs. Gynecologic Oncology) was made in terms of the proportion of procedures with high-power morcellation (Fig. 5). During the study period, 789 procedures with high-power morcellation (476 LSH, 313 laparoscopic myomectomies) were performed

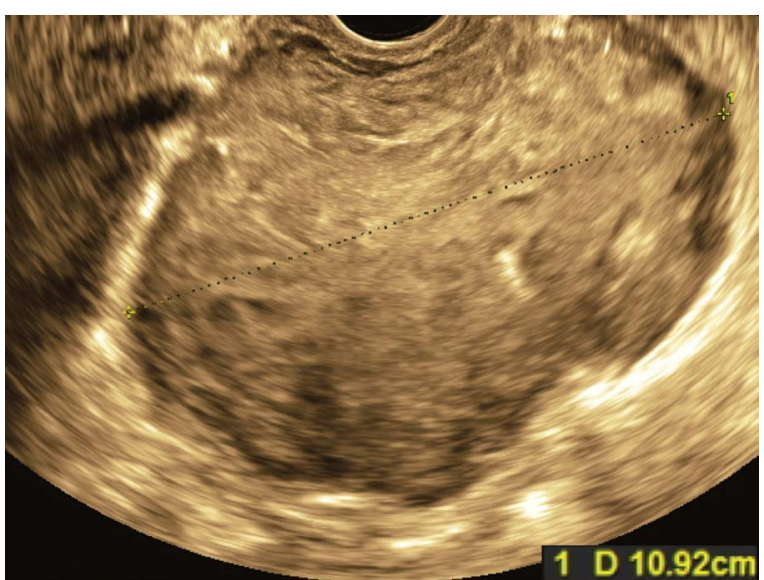

Figure 3. Presumed subserosal myoma in transvaginal ultrasound, which proved to be leiomyosarcoma in histopatologic examination

in the MIS unit, and no LMS was morcellated. Over the same time period, 162 high-power morcellations (111 LSH, 51 laparoscopic myomectomies) were carried out in the Gynecologic Oncology unit, where one case of LMS was morcellated during laparoscopic myomectomy (ratio of LMS morcellation, 1:162, $0.006, \mathrm{Cl} 0.001-0.034$; ratio of LMS morcellation on laparoscopic myomectomy, 1:51, 0.02, Cl 0.0035-0.1). 


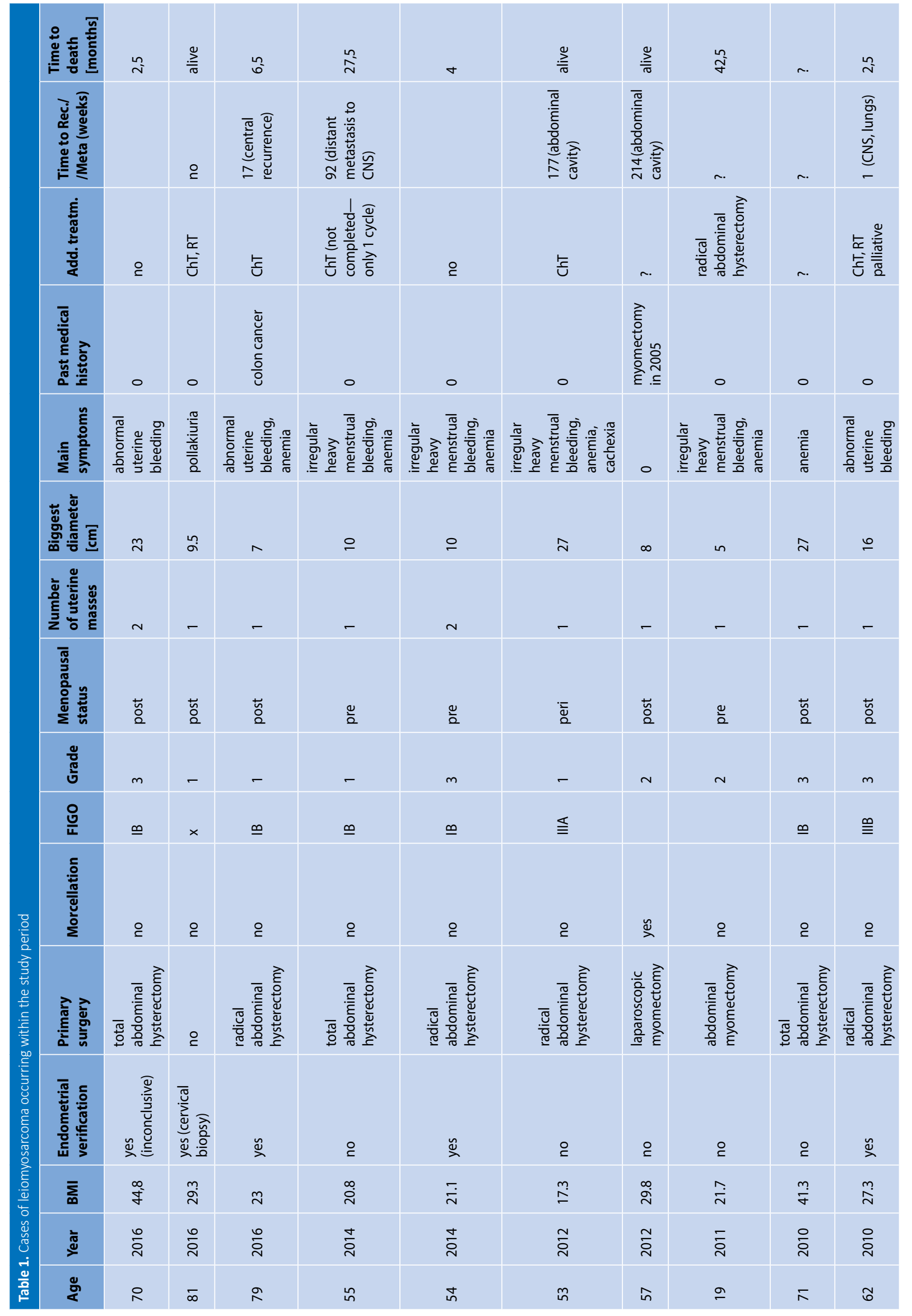




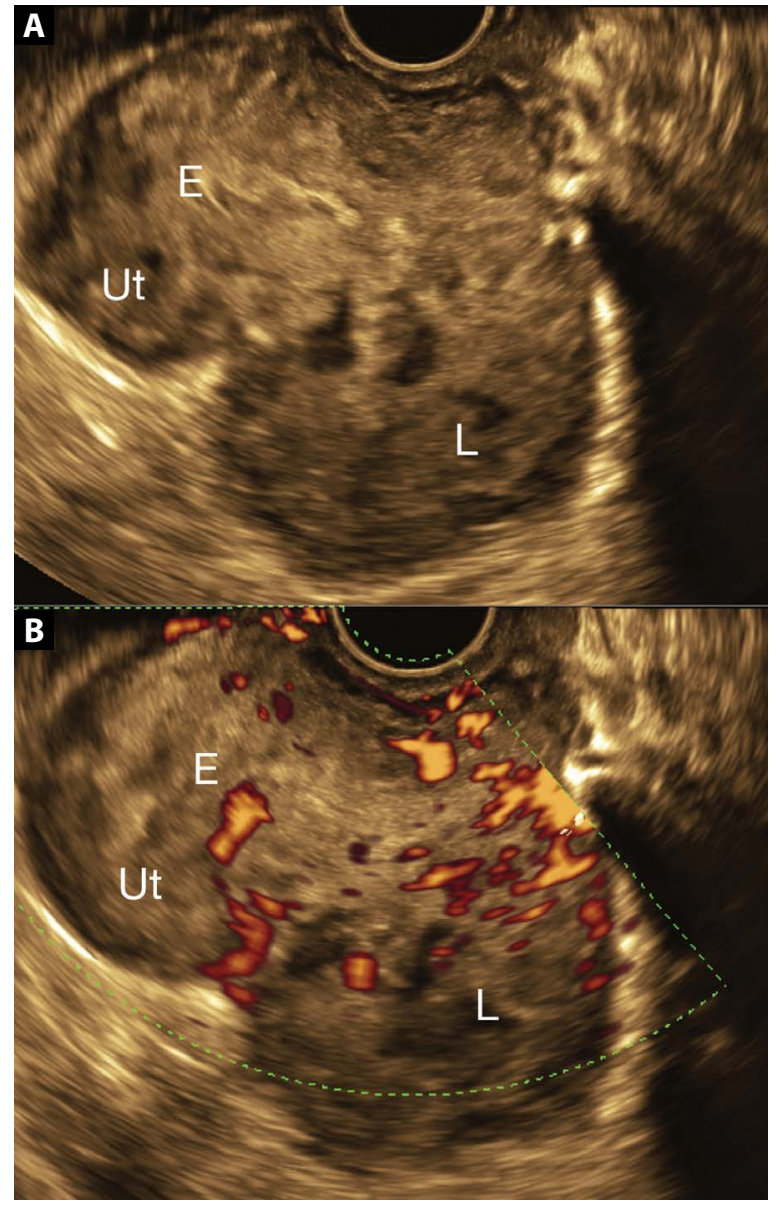

Figure 4. Uterus with presumed myoma. (A) Grey scale ultrasound, and (B) power Doppler. There are not highly specific features of leyomyosarcoma, which allow to accurate discrimination between myoma and LMS. Finaly, LMS was recognized after surgery $\mathrm{U}$ - uterus; $\mathrm{E}$ - endometrium; L — presumed myoma/LMS

\section{Abdominal vs. MIS surgery}

The incidence of occult LMS was $1: 1178(0.001 ; \mathrm{Cl} 0.0-$ $0.005)$ during MIS procedures and 1:1008 $(0.001 ; \mathrm{Cl} 0.0-$ 0.006 ) during laparoscopic procedures. Among 383 women treated by laparoscopic myomectomy, 364 underwent high-power morcellation (95\%; Fig. 1). Within this group, the one and only case of morcellated LMS was identified. No LMS was identified in patients from the LSH or hysteroscopic myomectomy groups.

The incidence of operating on occult LMS during traditional surgery was calculated and compared to the frequency during MIS. Five occult LMS cases were encountered on laparotomy. Given that the number of traditional approach procedures was 1497 , the estimated ratio for operating on occult LMS was 1:299 (0.003; Cl 0.0-0.015). This result, compared with the incidence calculated for MIS, represented a four-fold higher risk of occult LMS in traditional approach surgeries, but did not reach statistical significance (RR 3.9; $\mathrm{Cl} 0.46-33.6, \mathrm{P}=0.2)$.

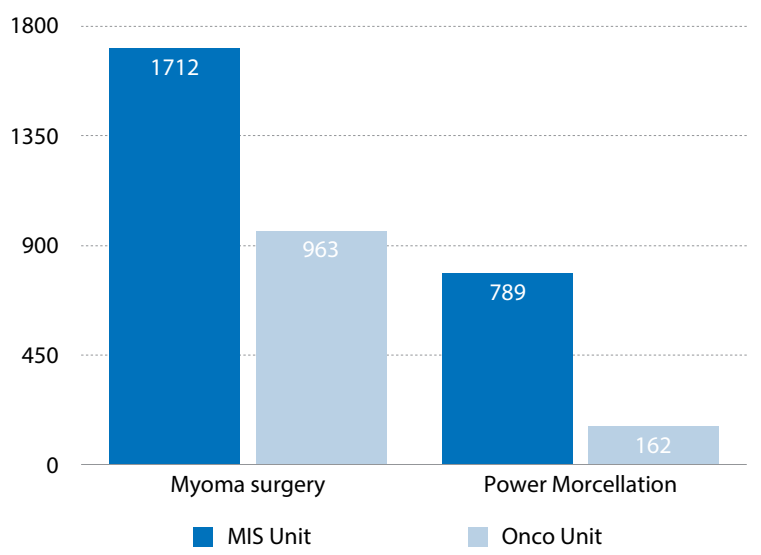

Figure 5. Myoma surgery and morcellation

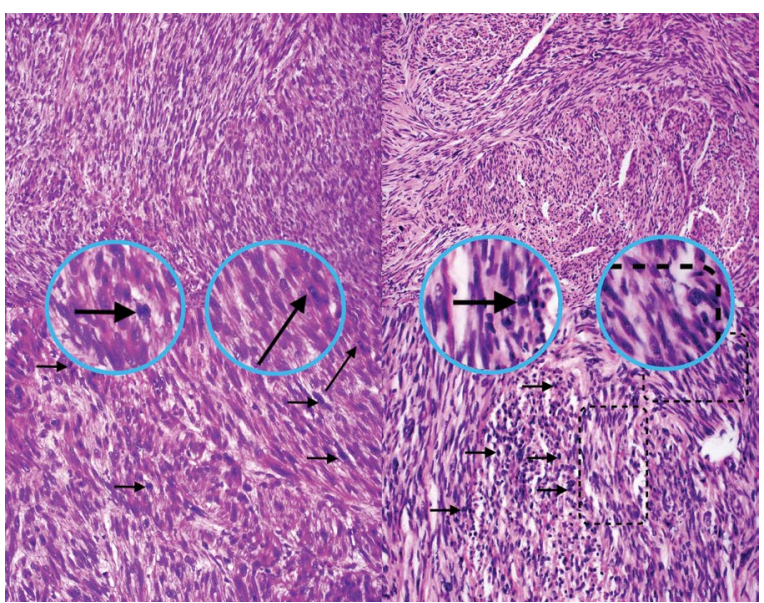

Figure 6. Surgical specimen of morcellated uterine leiomyosarcoma (left) and metastatic sarcoma of the greter omentum representing uterine leiomyosarcoma recurrence (right). Hematoxylin-eosin staining, (top row) 20x and (bottom row) 40x magnification. First circle - there are significant nuclear polymorphism (short arrows) and atypia of spindle tumor cells. Second circle - the number of mitotic figures reaches 14 on 10 high power fields (HPF) of view (40x) and the one is marked by the long arrow. Third circle - hypercellularity and nuclear atypia (arrows) and fourth circle - interlacing fascicles of spindle cells (marked with a dashed line), indicate malignancy

\section{Myomectomy in reproductive age}

Among 872 women who underwent myomectomy (mean age \pm SD: 37 years \pm 7.6 , median 37, lower-upper quartile: 32-42), 793 (91\%) were performed in women of reproductive age $(<45$ years). There was 1 case of LMS $(1: 793,0.001 ; \mathrm{Cl} 0.0-0.007)$.

\section{Case series of LMS: characteristic and surveillance}

The demographic and clinical data for women with LMS, both occult and preoperatively diagnosed cases, is shown in Table 1. Uterine LMS was morcellated by laparoscopic high-power morcellation during laparoscopic myomectomy in one 62-year-old woman. Apparently, no history of abnor- 
mal uterine bleeding, anemia, or bladder dysfunction was found in her medical records, and laparoscopic myomectomy was strongly preferred by patient to hysterectomy, as well as to the lack of treatment. Interestingly, the patient had already undergone conservative myomectomy 7 years earlier. The patient did not receive further treatment in our department, but was operated on in the general surgery ward of University Hospital 5 years after laparoscopy for abdominal tumor. Metastatic LMS of the greater omentum was confirmed histopathologically nearly 5 years after laparoscopic high-power morcellation (Fig. 6). As for other patients with occult LMS, one 19-year-old woman underwent abdominal myomectomy, and four postmenopausal patients underwent total abdominal hysterectomy as primary treatment.

Three women, aged 79, 54 and 62 years, were diagnosed with LMS on dilatation and curettage performed because of abnormal uterine bleeding/irregular heavy menstrual bleeding complicated by anemia. One 81-year-old woman was diagnosed by cervical biopsy.

\section{DISCUSSION}

The current study found that occult LMS occurred in 1:446 surgeries in women with presumed myomas, and more than half of all LMS cases recognized during the same period were occult. The incidence of LMS morcellation during laparoscopy was 1:951 procedures. The prevalence of occult LMS was higher in traditional abdominal procedures (1:299). Among the six women with occult LMS, five were peri- or postmenopausal, whereas one was a very young woman of reproductive age. Detailed analysis of abdominal versus MIS surgery and the Gynecologic Oncology unit versus the MIS unit indicated that an important factor influencing the rate of occult LMS was the preselection of patients with presumed myomas to abdominal surgery or MIS procedures, which could have minimized the rate of LMS morcellation. The results show that the use of morcellation for all suspected myomas may introduce a level of risk similar to both FDA calculations; $1: 498$ [4] and 1:495 to 1:1100 [13] of occult LMS. Paradoxically, in the MIS unit, with a significantly higher rate of power morcellation, the risk of encountering occult LMS was lower than in the gynecologic oncology unit due to the different prevalences of LMS (0:1712 vs 6:963, respectively). Thus, selection of specific centers and units (such as Gynecologic Oncology units) can overestimate the prevalence due to the higher-risk population treated. On the other hand, selected reports from only laparoscopic procedures or from only MIS centers may indicate a lower prevalence of occult LMS or risk of power morcellation [14-16] than multicenter studies [17].

The main limitations of this study are its retrospective nature and the single tertiary center study design. Strengths of the study include (i) analysis of data relative to all surgeries for presumed myomas during the study period, (ii) parallel analysis of the incidence of LMS recognized and treated during the study period, (iii) analysis was performed on data from the period, reflecting the current general (medium) quality of preoperative diagnostics (typical variability between different doctors, ultrasound machines and procedures), and (iv) the lack of influence of the FDA statement on the decision-making processes in the center (lack of specific time-dependent changes in diagnostics and procedures).

If our study design was based on a single MIS unit or MIS procedures only, we would have found no occult LMS cases as well as no occult LMS in women of reproductive age. Indeed, a recent case series of laparoscopic myomectomies in women of reproductive age reported no cases of LMS morcellation [16]. Based on these results and a recently suggested algorithm [18] for the use of power morcellation, one can suppose that morcellation in young women is very safe. In our series, abdominal myomectomy was performed in very young woman without suspicion of LMS. However, 9 of 10 women with LMS were peri- or postmenopausal and over 50 years of age. The FDA emphasizes [13] that sarcomas in women undergoing surgeries due to presumed myomas approaches $2-3 \%$ in those over 60 years [19-21]. It should be highlighted that leyomyosarcomas often are unidentified before surgery as showed the data from the Cancer Registry of Norway [22], and in the Norwegian cohort, power morcellation of LMS has not led to reduced survival. In our study the woman with LMS who underwent power morcellation is 1 of 3 women with the longest survival after LMS recognition from the case series. However, several new studies included in the recent FDA review [13] provide some evidence supporting an increased risk of relapse and decreased overall survival after power morcellation of LMS [19, 23, 24]. Conversely, another interpretation of the available data suggests that even intact removal of LMS has no advantage for mortality rate compared with power or any other type of morcellation [25]. Probably other multiple factors are more pivotal for patient survival than incidence of power morcellation.

The main implication of the current study is that the simple selection of patients through gynecological examination and ultrasonography may significantly change the prevalence of occult LMS and affect the risk associated with power morcellation. The incidence of occult LMS may be very low in MIS centers after appropriate preselection without significant risk of power morcellation. The FDA position after a recent review of the literature is the same as the previous [13], and power morcellators are contraindicated for removal of myomas in the majority of cases. From our European perspective, it is pivotal that well-balanced European Guidelines be developed by the main professional societies, considering advantages and disandvatages of these procedures in specific groups of women.

On the other hand, there is reasonable question whether morcellation of occult LMS is more risky than the delay 
of treatment by alternative therapies without histological examination (ie. magnetic-resonance-guided focused ultrasound surgery, uterine artery embolization) including pharmaceutical therapy (ie. ulipristal acetate) $[6,26]$.

Nowadays, studies should focus on improving the diagnostic accuracy and safety of myoma management. Probably the best way to obtain a true estimation of the incidence of LMS in presumed myoma cases and the morcellation rate of LMS is by performing a prospective registered multicenter study and maintaining an obligatory national registry of such events, as well as by reporting occult LMS cases that were unrecognized via histology before surgery for uterine tumors described as presumed myomas.

\section{CONCLUSIONS}

Selection of patients based on gynecological and ultrasound examinations may affect the prevalence of occult LMS in women with presumed myomas undergoing surgery and lower the risk of LMS morcellation. All women, even the very young, should be informed about the risk of occult LMS when the use of power morcellators or other conservative therapies are being considered, especially when histological examination is not being performed.

\section{Authors' roles}

A.L.: substantial contributions to study conception and design, data analysis and interpretation, article drafting and revision, and final approval of the version to be published. I.G.: substantial contributions to study conception and design; data collection, analysis and interpretation; article drafting and revision; and final approval of the version to be published. K.P.: substantial contributions to data interpretation, article drafting and revision, and final approval of the version to be published.

\section{Acknowledgments}

We thank Professor D. Adamek (Department of Pathomorphology, Jagiellonian University, Krakow, Poland) for their help in accessing the databases.

\section{Conflicts of interest}

This study received no funding, and the authors have no conflicts of interest to declare.

\section{REFERENCES}

1. Noorchashm H, Reed A. Tragedy, Trade-offs, and the Demise of Morcellation. New England Journal of Medicine. 2016; 374(26): 2605-2605, doi: $10.1056 /$ nejmc 1605896 .

2. Goff B. SGO not soft on morcellation: risks and benefits must be weighed. The Lancet Oncology. 2014; 15(4): e148, doi: 10.1016/s14702045(14)70075-0.

3. Patient safety must be a priority in all aspects of care. The Lancet Oncology. 2014; 15(2): 123, doi: 10.1016/s1470-2045(14)70042-7.

4. Food and Drug Administration (FDA). SpringerReference. , doi: $10.1007 /$ springerreference_32222.
5. Pritts E, Vanness D, Berek J, et al. The prevalence of occult leiomyosarcoma at surgery for presumed uterine fibroids: a meta-analysis. Gynecological Surgery. 2015; 12(3): 165-177, doi: 10.1007/s10397-015-0894-4.

6. Parker W, Berek J, Pritts E, et al. An Open Letter to the Food and Drug Administration Regarding the Use of Morcellation Procedures in Women Having Surgery for Presumed Uterine Myomas. Journal of Minimally Invasive Gynecology. 2016; 23(3): 303-308, doi: 10.1016/j.jmig.2015.12.012.

7. Gawron I, Skotniczny K, Ludwin A. Occult uterine malignancy during laparoscopic supracervical hysterectomy. Ginekologia Polska. 2018; 89(9): 467-474, doi: 10.5603/gp.a2018.0080.

8. Ludwin A, Ludwin I, Pityński K, et al. Transrectal Ultrasound-Guided Hysteroscopic Myomectomy of Submucosal Myomas With a Varying Degree of Myometrial Penetration. Journal of Minimally Invasive Gynecology. 2013; 20(5): 672-685, doi: 10.1016/j.jmig.2013.05.001.

9. Brown LD, Cai.TT, DasGupta A. Interval Estimation for a Binomial Proportion. Statistical Science. 2001;16(2): 101-133, doi: 10.1214/ss/1009213286, https://projecteuclid.org/euclid.ss/1009213286.

10. Daly LE. Confidence Limits Made Easy: Interval Estimation Using a Substitution Method. American Journal of Epidemiology. 1998; 147(8): 783-790, doi: 10.1093/oxfordjournals.aje.a009523.

11. Ashby D. Practical statistics for medical research. Douglas G. Altman, Chapman and Hall, London, 1991. Statistics in Medicine. 1991; 10(10): 1635-1636, doi: 10.1002/sim.4780101015.

12. Sheskin D. Handbook of Parametric and Nonparametric Statistical Procedures. Chapman and Hall. 2004, doi: 10.4324/9780203489536.

13. Food and Drug Administration, FDA Updated Assessment of The Use of Laparoscopic Power Morcellators to Treat Uterine Fibroids, $2017 \mathrm{https}: / \mathrm{www}$. fda.gov/downloads/medicaldevices/productsandmedicalprocedures/surgeryandlifesupport/ucm584539.pdf, doi: 10.1007/springerreference_32222.

14. Bojahr B, Wilde RDe, Tchartchian G. Malignancy rate of 10,731 uteri morcellated during laparoscopic supracervical hysterectomy (LASH). Archives of Gynecology and Obstetrics. 2015; 292(3): 665-672, doi: 10.1007/s00404-015-3696-z.

15. Vercellini $P, C$ ribiù $F$, Bosari $S$, et al. Prevalence of unexpected leiomyosarcoma at myomectomy: a descriptive study. American Journal of Obstetrics and Gynecology. 2016; 214(2): 292-294, doi: 10.1016/j.ajog.2015.09.092.

16. Pados G, Tsolakidis D, Theodoulidis V, et al. Prevalence of occult leiomyosarcomas and atypical leiomyomas after laparoscopic morcellation of leiomyomas in reproductive-age women. Human Reproduction. 2017; 32(10): 2036-2041, doi: 10.1093/humrep/dex258.

17. Sizzi O, Rossetti A, Malzoni M, et al. Italian multicenter study on complications of laparoscopic myomectomy. Journal of Minimally Invasive Gynecology. 2007; 14(4): 453-462, doi: 10.1016/j.jmig.2007.01.013.

18. Brölmann H, Tanos V, Grimbizis G, et al. Options on fibroid morcellation: a literature review. Gynecological Surgery. 2015; 12(1): 3-15, doi: 10.1007/s10397-015-0878-4.

19. Raine-Bennett T, Tucker LY, Zaritsky E, et al. Occult Uterine Sarcoma and Leiomyosarcoma. Obstetrics \& Gynecology. 2016; 127(1): 29-39, doi: 10.1097/aog.0000000000001187.

20. Mahnert N, Morgan D, Campbell D, et al. Unexpected Gynecologic Malignancy Diagnosed After Hysterectomy Performed for Benign Indications. Obstetrics \& Gynecology. 2015; 125(2): 397-405, doi: 10.1097/aog.0000000000000642.

21. Mao J, Pfeifer S, Zheng X, et al. Population-Based Estimates of the Prevalence of Uterine Sarcoma Among Patients With Leiomyomata Undergoing Surgical Treatment. JAMA Surgery. 2015; 150(4): 368, doi: 10.1001/jamasurg.2014.3518.

22. Skorstad M, Kent A, Lieng M. Preoperative evaluation in women with uterine leiomyosarcoma. A nationwide cohort study. Acta Obstetricia et Gynecologica Scandinavica. 2016; 95(11): 1228-1234, doi: 10.1111/aogs.13008.

23. George S, Barysauskas C, Serrano C, et al. Retrospective cohort study evaluating the impact of intraperitoneal morcellation on outcomes of localized uterine leiomyosarcoma. Cancer. 2014; 120(20): 3154-3158, doi: 10.1002/cncr.28844.

24. Gao Z, Li L, Meng Y. A Retrospective Analysis of the Impact of Myomectomy on Survival in Uterine Sarcoma. PLOS ONE. 2016; 11(2): e0148050, doi: 10.1371/journal.pone.0148050.

25. Pritts $\mathrm{E}$. The prevalence of occult leiomyosarcoma in women undergoing presumed fibroid surgery and outcomes after morcellation. Current Opinion in Obstetrics and Gynecology. 2017: 1, doi: 10.1097 /gco.0000000000000430.

26. Istre O. Unexpected Uterine Leiomyosarcoma During Laparoscopic Hysterectomy Treated 6 Months With Ulipristal Acetate and Contained Power Morcellation. Journal of Minimally Invasive Gynecology. 2017; 24(2): 198, doi: 10.1016/j.jmig.2016.08.004. 